\title{
Lentigo Maligna and Radiotherapy
}

TO THE EDITOR: Imiquimod has recently been shown to be effective in the management of lentigo maligna, as demonstrated in Images in Clinical Medicine (Nov. 17 issue) ${ }^{1}$ and in other reports. The authors correctly suggest that imiquimod may be effective for lentigo maligna, but readers are subsequently led to believe that perhaps this is the only alternative to surgery. Although imiquimod may be a promising nonsurgical alternative for the treatment of lentigo maligna, radiotherapy has been clinically successful, with excellent cosmesis. In one series of 101 patients with either lentigo maligna or lentigo maligna melanoma who were treated with radiotherapy and followed for a minimum of 2 years (mean, 8 years), the mean time to recurrence was 45.6 months, with a recurrence rate of 7 percent. ${ }^{2}$ Although radiotherapy may not be as convenient as treatment with a self-administered topical agent, it should be recognized as a potential therapeutic choice for patients with lentigo maligna. Radiotherapy can provide excellent cosmesis and local control.

Lynn D. Wilson, M.D., M.P.H.

Yale University School of Medicine

New Haven, CT 06510

lynn.wilson@yale.edu

1. Noel B, Kunzle N. Lentigo maligna. N Engl J Med 2005;353; 2176.

2. Farshad A, Burg G, Panizzon R, Drummer R. A retrospective study of 150 patients with lentigo maligna and lentigo maligna melanoma and the effect of radiotherapy using Grenz or soft X-rays. Br J Dermatol 2002;146:1042-6.
THE AUTHOR REPLIES: I agree with Dr. Wilson that radiotherapy is the most effective nonsurgical treatment for lentigo maligna. ${ }^{1}$ However, imiquimod is an emerging new treatment that has many advantages. It can be administered by the patient, reducing the cost of treatment. Side effects, such as skin irritation, are transient and resolve spontaneously after treatment, leaving no scars or skin marks. By contrast, radiotherapy may induce chronic radiodermatitis and, sometimes, skin cancer. ${ }^{2}$ Imiquimod not only stimulates the immune system but also inhibits angiogenesis, a fact that contributes to its clinical efficacy against different types of dermatologic conditions such as genital warts, actinic keratoses, lentigo maligna, basal-cell carcinoma, and squamous-cell carcinoma. ${ }^{3}$

Bernard Noël, M.D.

Centre Hospitalier Universitaire Vaudois

1011 Lausanne, Switzerland

bernard.noel@chuv.ch

1. Zalaudek I, Horn M, Richtig E, Hodl S, Kerl H, Smolle J. Local recurrence in melanoma in situ: influence of sex, age, site of involvement and therapeutic modalities. Br J Dermatol 2003; 148:703-8.

2. Suter L. Long-term (chronic, late) radiation reactions of the skin. In: Panizzon RG, Cooper JS, eds. Radiation treatment and radiation reactions in dermatology. Berlin: Springer-Verlag, 2004: 143-57.

3. Hengge UR, Schaller J. Successful treatment of invasive squamous cell carcinoma using topical imiquimod. Arch Dermatol 2004;140:404-6. [Erratum, Arch Dermatol 2005;141:764.]

\section{Treatment for Rheumatic Disorders}

TO THE EDITOR: With regard to the letter by Dr. April (Oct. 6 issue), ${ }^{1}$ in reference to a Perspective article by Topol on the overuse of nesiritide for congestive heart failure (July 14 issue) $)^{2}$ : Dr. April claims that the current president of the American College of Rheumatology (ACR), along with an unnamed past president, encouraged the use of intravenous office infusion of infliximab (Remicade) over other treatments, such as self-injected medications, "as a way to offset poor reimburse- ment for patient care." We are writing to respond to Dr. April's unfounded statements.

The issue of access to medications for the treatment of rheumatoid arthritis and other autoimmune diseases came into focus with the development of new biologic treatments that are administered only by self-injection or as intravenous infusions. At the time that these treatments were introduced, Medicare did not cover selfinjected medications, which forced many patients 
to choose infusions administered in hospitals or in physicians' offices. Since 2002, the ACR has advocated that patients should have access to all approved therapies for rheumatic disease and that decisions about which treatment is most appropriate should be made by patients and their physicians. The ACR has also clearly stated that rheumatologists should be compensated for the work that they do and not for the drugs that they choose. We believe that the only factor that should influence a physician's decision regarding which medication to prescribe is the best interest of the patient.

The ACR leadership, including the current president (Dr. Tindall), fought for the inclusion of self-injectable medications in the 2003 Medicare Replacement Drug Demonstration Project and worked hard to encourage rheumatologists to help their patients participate in this project. As of October 7, 2005, 40,239 patients were en- rolled, with coverage for self-injected medications, including 14,337 patients with rheumatoid arthritis. Neither the ACR nor its president has ever encouraged physicians to favor office infusions over other treatments for rheumatic diseases.

Elizabeth A. Tindall, M.D.

Oregon Health and Science University Portland, OR 97224

David A. Fox, M.D.

University of Michigan Medical Center Ann Arbor, MI 48109

Dr. Tindall reports having served as an investigator for Amgen, Bristol-Myers Squibb, Genentech, and Abbott. Dr. Fox reports having received a research grant from Genentech, and his institution has received educational grants from Abbott, Amgen, Centocor, and Wyeth.

1. April PA. Nesiritide - not verified. N Engl J Med 2005; 353:1526.

2. Topol EJ. Nesiritide - not verified. N Engl J Med 2005; 353:113-6.

\section{Antibodies Neutralizing Peginterferon Alfa during Retreatment of Hepatitis C}

TO THE EDITOR: Peginterferons are widely used to treat chronic hepatitis $\mathrm{C}$ virus (HCV) infection, an important cause of liver cirrhosis and hepatocellular carcinoma. ${ }^{1-4}$ We describe a patient who did not have a virologic response to peginterferon and had a virtual absence of circulating peginterferon alfa-2a but had neutralizing antibodies against interferon alfa.

A 38-year-old man with liver cirrhosis (ChildPugh class A; score, 5) was referred to our hospital for evaluation of his chronic HCV infection. He had had no response to a 16-week course of treatment with peginterferon alfa-2b and ribavirin during the previous year. We enrolled him in an 18-month study of high-dose peginterferon alfa-2a (at a dose of $360 \mu \mathrm{g}$ per week during the first 4 weeks) in combination with ribavirin (at a dose based on weight). The study enrolled patients with genotype 1 , and the index patient was the second of nine patients to enroll. The study was approved by our institutional review board, and patients provided written informed consent. At the start of treatment, the index pa- tient's serum level of alanine aminotransferase was 106 IU per milliliter (normal, <41), and the serum level of HCV RNA was 6.8 log copies per milliliter.

To determine the effect of the administration of peginterferon alfa-2a on pharmacokinetic and plasma viral kinetic profiles, peginterferon alfa$2 \mathrm{a}$ levels were measured with the use of a quantitative sandwich enzyme-linked immunosorbent assay (ELISA) for interferon alfa (Bender MedSystems Diagnostics GmbH), and HCV RNA levels were quantitated (Cobas Amplicor HCV test, Roche Diagnostics) at baseline and at days 1, 2, $4,7,14,21$, and 28 after the start of treatment. We observed a variation in pharmacokinetic profiles among patients. In contrast to the other eight patients, the index patient's levels of peginterferon alfa-2a were virtually undetectable, and he did not have any virologic response to treatment (Fig. 1A and 1B). This finding led us to measure the level of specific antibodies to interferon alfa by ELISA. Interferon alfa-specific antibodies were detected at low levels before the 